\title{
Myasthenia and Pregnancy: About one Case
}

\author{
R. El Hadrami", Z. Abjaw, H. Ribahi, A-R EL ADIB
}

Maternity Intensive Care Unit, Mother-Child Hospital, Mohamed VI University Hospital Center, Marrakech, Morocco

\author{
DOI: $10.36348 /$ sijog.2020.v03i01.004 | Received: 01.01.2020 | Accepted: 08.01.2020 | Published: 30.01 .2020 \\ *Corresponding author: Reda El Hadrami
}

\section{Abstract}

Myasthenia gravis (MG) is an autoimmune disease of the neuromuscular junction affecting mainly young women, on this poster reporting a clinical case of a parturient parturient of 21 years old, primiparous, diagnosed myasthenia for 6 years, thymectomized, under corticosteroid therapy, azathioprine and mytelase, who presents for a pregnancy of $36 \mathrm{SA}+3$ without signs of muscle weakness. The delivery was carried out vaginally under epidural analgesic giving birth to a newborn of $2 \mathrm{~kg} 800$, male, Apgar a 4/4/4, lethargic with congenital myasthenia who died after 10 hours of life. The diaper suites were simple at day 4 of the postpartum the parturient presented a serious myasthenic crisis at the paraclinical exploration the biological assessment showed a slight inflammatory syndrome the therapeutic approach consisted in a conditioning of the patient, corticosteroid, prostigmine, azathioprine, mytelase, as well as a cure of 5 days of immunoglobulin. The evolution was favorable at the end of the 3rd day and the patient was transferred to the neurology department on the 6th day and left home at the end of the 10th day.

Keywords: pregnancy, myasthenia gravis, myasthenia neonatal, thymectomy.

Copyright @ 2020: This is an open-access article distributed under the terms of the Creative Commons Attribution license which permits unrestricted use, distribution, and reproduction in any medium for non-commercial use (NonCommercial, or CC-BY-NC) provided the original author and source are credited.

\section{INTRODUCTION}

Myasthenia gravis (MG) is an autoimmune disorder of the neuromuscular junction, affecting mainly young women, resulting in progressive paresis caused by antibodies attacking cholinergic receptors.

The prevalence of MG is between 1/10000 and $1 / 50000$, of which $2 / 3$ are women, usually during their fertility period: 2 nd and 3 rd decades of life $[1,2]$

In case of MG, the parturient and the newborn are likely to develop, in a variable way, weaknesses of the skeletal muscles.

On this paper, we report the case of a MG, as well as a review of the literature in order to identify the diagnostic and therapeutic strategies including the management of childbirth and the management of the neonatal period.

\section{CASE REPORT}

We report the case of a 21-year-old parturient, diagnosed as myasthenic for 6 years, thymectomized, under steroids, azathioprine and mytelase, is presented for 36 weeks and 3 days pregnancy without signs of muscle weakness. The delivery was performed vaginally under epidural analgesic giving birth to a $2 \mathrm{~kg} 800$ male newborn, with an Apgar score at 4/4/4, lethargic having congenital myasthenia gravis, he died 10 hours after birth. There were no complications following childbirth.

Four days after childbirth the parturient presented a serious myasthenic crisis made of generalized muscular weakness ( $3 / 5$ to the 4 limbs), a bilateral ptosis, as well as disorders of swallowing and phonation. Myasthenia gravis score was at 35/100.

At the paraclinical exploration the biological report showed a slight inflammatory syndrome (CRP 36 $\mathrm{mg} / \mathrm{dL}$ (normal value $<5 \mathrm{mg} / \mathrm{dL}$ ), $12000 / \mu \mathrm{L}$ of leucocytes (normal value $4000-9400 / \mu \mathrm{L}$ ) without image of pneumopathy from inhalation on chest x-ray.

After multidisciplinary consultation, the therapeutic approach consisted on a conditioning of the patient (Oxygen at a rate of $2 \mathrm{~L} / \mathrm{min}$, installation of a naso-gastric tube, session of VNI of one hour each 4 
R. El Hadrami et al; Sch Int J Obstet Gynec, Jan. 2020; 3(1): 11-13

hours), corticosteroid, prostigmine, imurel, mytelase, as well as a 5-day of immunoglobulin treatment.

The evolution was favorable at the end of the 3 rd day (recovery of muscular forces at $4 / 5$, regression of ptosis and phonation disorders) and walking and feeding became possible on the 5 th day

The patient was transferred to the neurology department on the 6th day and discharged at home after 10 days.

\section{DISCUSSION}

The evolution of MG and its influence on pregnancy is unpredictable, indeed in $31 \%$ myasthenic parturients remain stable during pregnancy, $29 \%$ note a regression of symptoms, and $40 \%$ show an exacerbation of their disease [1].

Exacerbation of MG can occur at any stage of pregnancy, especially during the first trimester and first month after childbirth [3-5].

Otherwise, the evolution of MG during previous pregnancies does not make it possible to predict the evolution during subsequent pregnancies [5].

MG has little effect on the evolution of pregnancy, the incidence of spontaneous abortion and intrauterine growth retardation remains unchanged [5] However, we note in the literature an increase in the frequency of premature rupture of membranes $[5,6]$

In pre-eclampsia (PE), there has been no increase in its incident but cases have already been described [7]. It should be kept in mind when MG and $\mathrm{PE}$ are combined magnesium sulfate is contraindicated.

In general, myasthenic parturients should be closely monitored during their pregnancy, including not only the obstetrician but also the neurologist, some authors suggest that obstetrical ultrasound should be frequently performed to detect fetal akinesia manifested by a reduction of fetal movements and respiratory movements which can induce a hydramnios [8].

The risk of hypoventilation in the myasthenic parturient is to be watched in view of the limitation of the diaphragmatic stroke by the pregnant uterus, and any respiratory decompensation must be detected and treated urgently after multidisciplinary consultation.

Several approaches and therapeutic strategies should be adopted in parturients with MG. Passing firstly by the information of the parturient of the course of the pregnancy and the maternal and fetal risks that the pregnancy presents follows the education of the parturient so that she avoids the useless physical efforts and thus to avoid the weakness muscular.
Thymectomy is recommended for the treatment of myasthenia gravis, and appears to reduce the incidence of exacerbations compared to nonthymectomized patients [9] however there is no difference between the two groups in the incidence of neonatal MG

Medical treatment of myasthenia gravis should not be stopped during pregnancy and even seems safe especially anti-cholinesterase inhibitors [3], only a few cases have reported neonatal $\mathrm{MG}$ associated with microcephaly [10]

Corticosteroid therapy also seems safe during pregnancy, with a low teratogenic risk [11, 12]; there has been a slight increase in cleft lip and palate in children from mothers taking corticosteroids [13]. However, the risk of premature rupture of the membranes is increased in parturients with high doses of corticosteroids [14]

Azathioprine, meanwhile, has not been recommended during pregnancy since it exposes the fetus to bone marrow failure [15].

Regarding the modalities of delivery, the vaginal approach seems preferred by the authors since auto antibodies do not affect the uterus [3, 16], however, since the second part of the work requires the striated pelvic muscles, the delivery may be facilitated by an instrumentalisation. Caesarean section will only be performed if the obstetric indication is asked [3]. Regarding the analgesic component, the epidural is preferred to morphine and muscle relaxant which are contraindicated

Neonatal MG has been observed in only 10 to $20 \%$ of newborns born to myasthenic mothers $[17,18]$. Neuromuscular symptomatology usually manifests during the first 12 to 48 hours of life as the value of close monitoring of the newborn during this period [18]. Generally, there is no correlation between the severity of the maternal form and the occurrence of MG in the newborn $[3,17]$

Nevertheless, cases of pulmonary hypoplasia, congenital arthrogryposis, and non-specific hyperbilirubinemia have been reported $[3,19,20]$. In spite of the rarity of these complications the parturients carrying myasthenia, even stabilized, must be informed of this risk.

\section{CONCLUSION}

Parturients with myasthenia gravis are considered high-risk pregnancies, given the unpredictable course of their disease during pregnancy as well as the risk of muscle weakness and especially maternal and neonatal respiratory failure, hence the interest of a multidisciplinary follow-up. 
R. El Hadrami et al; Sch Int J Obstet Gynec, Jan. 2020; 3(1): 11-13

\section{REFERENCES}

1. Vincent, A., McConville, J., Farrugia, M. E., \& Newsom-Davis, J. (2004, March). Seronegative myasthenia gravis. In Seminars in neurology. Copyright $\odot 2004$ by Thieme Medical Publishers, Inc., 333 Seventh Avenue, New York, NY 10001, USA. 24(01), 125-133

2. Plauché, W. C. (1991). Myasthenia gravis in mothers and their newborns. Clinical obstetrics and gynecology, 34(1), 82-99.

3. Djelmis, J., Sostarko, M., Mayer, D., \& Ivanisevic, M. (2002). Myasthenia gravis in pregnancy: report on 69 cases. European Journal of Obstetrics \& Gynecology and Reproductive Biology, 104(1), 2125.

4. Plauché, W. C. (1991). Myasthenia gravis in mothers and their newborns. Clinical obstetrics and gynecology, 34(1), 82-99.

5. Batocchi, A. P., Majolini, L., Evoli, A., Lino, M. M., Minisci, C., \& Tonali, P. (1999). Course and treatment of myasthenia gravis during pregnancy. Neurology, 52(3), 447-447.

6. Hoff, J. M., Daltveit, A. K., \& Gilhus, N. E. (2003). Myasthenia gravis: consequences for pregnancy, delivery, and the newborn. Neurology, 61(10), 1362-1366.

7. Duff, G. B. (1979). Preeclampsia and the patient with myasthenia gravis. Obstetrics and gynecology, 54(3), 355-358.

8. Stoll, C., Ehret- Mentre, M. C., Treisser, A., \& Tranchant, C. (1991). Prenatal diagnosis of congenital my asthenia with arthrogryposis in a myasthenic mother. Prenatal diagnosis, 11(1), 1722.

9. Eden, R. D., \& Gall, S. A. (1983). Myasthenia gravis and pregnancy: a reappraisal of thymectomy. Obstetrics and gynecology, 62(3), 328-333.

10. Niesen, C. E., \& Shah, N. S. (2000). Pyridostigmine-induced microcephaly. Neurology, 54(9), 1873-1874.

11. Fraser, F. C., \& Sajoo, A. (1995). Teratogenic potential of corticosteroids in humans. Teratology, 51(1), 45-46.
12. Czeizel, A. E., \& Rockenbauer, M. (1997). Population- based case- control study of teratogenic potential of corticosteroids. Teratology, 56(5), 335-340.

13. Rodríguez- Pinilla, E., \& Luisa Martínez- Frías, M. (1998). Corticosteroids during pregnancy and oral clefts: a case- control study. Teratology, 58(1), 2-5.

14. Pickrell, M. D., Sawers, R., \& Michael, J. (1988). Pregnancy after renal transplantation: severe intrauterine growth retardation during treatment with cyclosporin A. British medical journal (Clinical research ed.), 296(6625), 825.

15. DeWitte, D. B., Buick, M. K., Cyran, S. E., \& Maisels, M. J. (1984). Neonatal pancytopenia and severe combined immunodeficiency associated with antenatal administration of azathioprine and prednisone. The Journal of pediatrics, 105(4), 625628.

16. Klehmet, J., Dudenhausen, J., \& Meisel, A. (2010). Course and treatment of myasthenia gravis during pregnancy. Der Nervenarzt, 81(8), 956-962.

17. Donaldson, J. O., Penn, A. S., Lisak, R. P., Abramsky, O., Brenner, T., \& Schotland, D. L. (1981). Antiacetylcholine receptor antibody in neonatal myasthenia gravis. American Journal of Diseases of Children, 135(3), 222-226.

18. Bartoccioni, E., Evoli, A., Casali, C., Scoppetta, C., Tonali, P., \& Provenzano, C. (1986). Neonatal myasthenia gravis: clinical and immunological study of seven mothers and their newborn infants. Journal of neuroimmunology, 12(2), 155161.

19. Vincent, A., Newland, C., Beeson, D., Riemersma, S., Newsom-Davis, J., Brueton, L., \& Huson, S. M. (1995). Arthrogryposis multiplex congenita with maternal autoantibodies specific for a fetal antigen. The Lancet, 346(8966), 24-25.

20. Daskalakis, G. J., Papageorgiou, I. S., Petrogiannis, N. D., Antsaklis, A. J., \& Michalas, S. K. (2000). Myasthenia gravis and pregnancy. European Journal of Obstetrics \& Gynecology and Reproductive Biology, 89(2), 201-204. 